\title{
A Biosynthetic Scaffold that Facilitates Chondrocyte-Mediated Degradation and Promotes Articular Cartilage Extracellular Matrix Deposition
}

\author{
Balaji V. Sridhar ${ }^{1,2}$ • Eric A. Dailing ${ }^{1}$ J. Logan Brock ${ }^{1,2}$ • Jeffrey W. Stansbury ${ }^{1,3}$ • \\ Mark A. Randolph ${ }^{4,5}$ - Kristi S. Anseth ${ }^{1,2,6}$
}

Received: 11 April 2015 / Accepted: 30 July 2015 /Published online: 30 September 2015

(C) The Regenerative Engineering Society 2015

\begin{abstract}
Articular cartilage remains a significant clinical challenge to repair because of its limited self-healing capacity. Interest has grown in the delivery of autologous chondrocytes to cartilage defects, and combining cell-based therapies with scaffolds that capture aspects of native tissue and allow cell-mediated remodeling could improve outcomes. Currently, scaffold-based therapies with encapsulated chondrocytes permit matrix production; however, resorption of the scaffold often does not match the rate of matrix production by chondrocytes, which can limit functional tissue regeneration. Here, we designed a hybrid biosynthetic system consisting of poly(ethylene glycol) (PEG) endcapped with thiols and crosslinked by norbornenefunctionalized gelatin via a thiol-ene photopolymerization.
\end{abstract}

Electronic supplementary material The online version of this article (doi:10.1007/s40883-015-0002-3) contains supplementary material, which is available to authorized users.

Kristi S. Anseth

kristi.anseth@colorado.edu

1 Department of Chemical and Biological Engineering, University of Colorado, Boulder, CO, USA

2 BioFrontiers Institute, University of Colorado, Boulder, CO, USA

3 Department of Craniofacial Biology, School of Dental Medicine, University of Colorado, Aurora, CO, USA

4 Department of Orthopedic Surgery, Laboratory for Musculoskeletal Tissue Engineering, Massachusetts General Hospital, Harvard Medical School, Boston, MA, USA

5 Plastic Surgery Research Laboratory, Division of Plastic Surgery, Massachusetts General Hospital, Harvard Medical School, Boston, MA, USA

6 Howard Hughes Medical Institute, University of Colorado, Boulder, CO, USA
The protein crosslinker was selected to facilitate chondrocytemediated scaffold remodeling and matrix deposition. Gelatin was functionalized with norbornene to varying degrees $(\sim 4$ 17 norbornenes/gelatin), and the shear modulus of the resulting hydrogels was characterized $(<0.1-0.5 \mathrm{kPa})$. Degradation of the crosslinked PEG-gelatin hydrogels by chondrocytesecreted enzymes was confirmed by gel permeation chromatography. Finally, chondrocytes encapsulated in these biosynthetic scaffolds showed significantly increased glycosaminoglycan deposition over just 14 days of culture, while maintaining high levels of viability and producing a distributed matrix. These results indicate the potential of a hybrid PEG-gelatin hydrogel to permit chondrocytemediated remodeling and promote articular cartilage matrix production. Tunable scaffolds that can easily permit chondrocyte-mediated remodeling may be useful in designing treatment options for cartilage tissue engineering applications.

\section{Lay Summary}

Articular cartilage remains a significant clinical challenge to repair because of its limited self-healing capacity. In this manuscript, a biosynthetic scaffold crosslinked by both gelatin and poly(ethylene glycol) (PEG) was developed to encapsulate primary cartilage cells, also known as chondrocytes. This hybrid scaffold facilitated cartilage-specific extracellular matrix (ECM) molecule deposition by permitting cell-mediated, localized degradation of the construct so that encapsulated chondrocytes had pericellular space to generate tissue. Furthermore, the data show that the mechanical properties of this gel can be easily modified and this system can be formed in situ at a defect site via a photopolymerizable reaction. The results of this manuscript indicate the potential of this novel system in designing treatment options for cartilage tissue regeneration applications. 
Keywords Cartilage tissue engineering - Chondrocytes . Gelatin · Local degradation $\cdot$ Hydrogel $\cdot$ Photopolymerization

\section{Introduction}

Articular cartilage has limited self-healing properties, which can necessitate clinical interventions to heal tissue defects. Chondrocytes, the sole, differentiated resident cells found in mature articular cartilage, are responsible for the generation and maintenance of tissue extracellular matrix (ECM) [1]. When combined with encapsulated chondrocytes, biofunctional scaffolds can facilitate cartilage ECM production and deposition. A variety of natural and synthetic materials have been examined as potential cell carriers and as therapeutic solutions for cartilage repair [2-5].

A limitation with many of the currently studied chondrocyte scaffold carriers is that their resorption rates do not match the rate of matrix deposition by encapsulated cells as found in native tissue [6]. Synthetic hydrogel carriers often limit deposition of chondrocyte-secreted matrix molecules to the space around the cell (i.e., the pericellular space) [7, 8]. To overcome this issue, hydrogels have been engineered to hydrolytically degrade at physiological $\mathrm{pH}$, and while bulk degradation can be readily controlled, numerous material properties are highly coupled to this degradation. For example, high extents of degradation must occur before an ECM protein, like collagen, can assemble throughout hydrogel scaffolds, but this often coincides with a precipitous drop in gel mechanics [7,9]. Alternatively, natural ECM proteins (e.g., collagen and hyaluronan) can form fibrillar hydrogel networks and provide numerous biological cues to guide tissue deposition by encapsulated cells [10]. These ECM proteins can also be easily degraded by encapsulated cells, which leads to a cell-mediated, local degradation mechanism [11]. However, natural protein-derived scaffolds are often mechanically weak, and it is difficult to control their reproducibility and degradation, which can necessitate synthetic modification to these materials to control their time-varying properties as well as facilitate the cell encapsulation process [12-14]. As a result, recent efforts in the field have included a focus on hybrid synthetic ECM mimics that have the potential to capture the tunability of synthetic scaffolds while integrating the properties of a celldictated degradation.

Previous work in our group demonstrated that cartilage cells encapsulated in poly(ethylene glycol) (PEG) hydrogels crosslinked by a collagen-derived peptide sequence (KCGPQG $\downarrow$ IWGQCK) generated constructs with increased, widespread articular cartilage-specific ECM compared to nondegradable gels [15]. These findings supported the hypothesis that local, cell-mediated degradation not only promotes cartilage tissue deposition but also maintains and in some cases increases scaffold mechanical integrity, in contrast to the decrease in bulk modulus typically found in hydrolytically cleavable scaffolds. However, it was found that enzymes secreted by encapsulated chondrocytes alone could not cleave the collagen-derived peptide linker with appropriate kinetics to enable a widespread distribution of matrix macromolecules. In fact, those constructs had the same pericellular matrix deposition pattern found in nondegradable scaffolds. Although previously, the Hubbell group engineered the collagenderived peptide linker GPQG $\downarrow$ IWGQ to be more responsive to matrix metalloproteinases (MMPs) [16], chondrocytes were found to remodel synthetic scaffolds more appropriately in the presence of cartilage progenitor cells, mesenchymal stem cells (MSCs). Only when encapsulated in coculture with MSCs could the relatively metabolically inactive chondrocytes [17] readily degrade the sequence and then generate ECM throughout the scaffold. Furthermore, others have shown that cellsecreted MMPs are able to cleave a full-length protein at a greater rate than a peptide derived from that protein [18]. Collectively, these findings motivated the experiments reported herein, where we investigate whether a hybrid scaffold composed of PEG and a full-length protein, with a larger amount of collagenase-cleavable sequences per molecule than a peptide, would be responsive to chondrocyte-mediated local degradation and permit widespread matrix deposition.

Hybrid scaffolds that combine full-length proteins with synthetic linkers have been widely explored in tissue engineering applications. For example, fibrinogen [19-21] and collagen [22-24] have been chemically modified to allow covalent attachment to PEG by controlled reactions and thereby facilitate encapsulated cell development and proliferation. Collagen appears to be a good candidate material to use as a scaffold with chondrocytes since degradation of collagen is a rate-limiting step in cartilage remodeling [25]. However, collagen is resistant to most proteases and requires special collagenases for its enzyme hydrolysis. On the other hand, gelatin, a natural biomacromolecule derived from denatured collagen, is less antigenic, more cost-effective [26], and susceptible to more proteases than collagen [27]. These attributes potentially make gelatin an easier substrate to cleave by chondrocytesecreted enzymes. Chondrocytes are also known to secrete gelatinases [28, 29], which can specifically cleave gelatin more efficiently than most proteases. Finally, gelatin has been successfully employed as a scaffold to promote articular cartilage-specific matrix production of encapsulated chondrocytes [30, 31], and hybrid PEG-gelatin networks have been developed for other tissue engineering applications with high cell viability under photopolymerization conditions [32-34].

In this work, we report the development of a gelatin network crosslinked with PEG for use with encapsulated chondrocytes and observe that the full-length protein is sensitive to local degradation cues and facilitates widespread cartilage-specific ECM deposition. Specifically, gelatin was 
modified to contain pendant norbornene functionalities and reacted via a facile thiol-ene photopolymerization with PEG dithiols. The photoinitiated thiol-ene reaction is fast and highly efficient and permits precise spatial and temporal control of network formation [35].

\section{Materials and Methods}

\section{Functionalization of Gelatin with Norbornene}

Gelatin type A 300 bloom ( $1 \mathrm{wt} \%(w / v))$ with $M_{n} \sim 75 \mathrm{kDa}$ [36] (Sigma-Aldrich), which contains around 21 primary amines per molecules, was dissolved in $\mathrm{pH} 8.5$ sodium bicarbonate. 5-Norbornene-2-acetic acid succinimidyl ester (Sigma-Aldrich) was added to the gelatin solution (21 molar eq. to gelatin for 1:1 norbornene (NB):gelatin amine stoichiometric ratio, 10.5 molar eq. to gelatin for 0.5:1 NB:gelatin amine ratio, and 5.25 molar eq. to gelatin for $0.25: 1$ NB:gelatin amine ratio) and reacted with free amines on the gelatin molecule at $37{ }^{\circ} \mathrm{C}$ for $1 \mathrm{~h}$. The functionalized gelatin was dialyzed against $\mathrm{pH} 8.5$ sodium bicarbonate for $4 \mathrm{~h}$ at RT exchanging buffer every hour, using Slide-A-Lyzer ${ }^{\mathrm{TM}} \mathrm{G} 2$ dialysis cassette MW $10 \mathrm{kDa}$ (Thermo Scientific). After dialysis, solutions were frozen, lyophilized, and stored at $-20{ }^{\circ} \mathrm{C}$ until use.

The degree of functionalization of gelatin with norbornene was quantified as previously described [32]. Briefly, the lysyl residue modification of gelatin was evaluated via the trinitrobenzenesulfonicacid assay (TNBSA, Thermo Scientific), which is a colorimetric assay that involves reacting the TNBSA reagent with primary amines on proteins for $2 \mathrm{~h}$ at $37^{\circ} \mathrm{C}$, stopping the reaction with $10 \%$ SDS and $1 \mathrm{~N} \mathrm{HCl}$, and then reading the absorbance at $\lambda=335 \mathrm{~nm}$ using a Synergy $\mathrm{H} 1$ microplate reader (BioTek). The functionalization efficiency was calculated using

$$
\left(1-\frac{[\text { amines }] \text { after gelatin modification }}{[\text { amines }] \text { before gelatin modification }}\right) \times 100 \%
$$

\section{Characterization of Degradation of the Functionalized Gelatin}

To assess enzymatic cleavage of the norbornenefunctionalized gelatin, $0.2 \mathrm{wt} \%$ gelatin solutions with varying extents of functionalization $(1: 1,0.5: 1$, and $0.25: 1 \mathrm{NB}$ :gelatin amine molar ratios or unmodified gelatin) were dissolved in $0.1 \mathrm{M}$ sodium nitrate (Sigma-Aldrich) and $0.1 \mathrm{M}$ sodium dibasic phosphate (Sigma-Aldrich), so that the resulting solution could be assessed using aqueous mobile phase gel permeation chromatography (GPC) as previously described [37]. The gelatin solutions were incubated with either 20 units $/ \mathrm{mL}$ $(\sim 0.1 \mathrm{mg} / \mathrm{mL})$ type II collagenase (Worthington Biochemical) or chondrocyte-conditioned media from cells cultured for 3 days. The enzyme solutions were reacted with the functionalized gelatin for $1 \mathrm{~h}$ at $37{ }^{\circ} \mathrm{C}$. GPC was performed using a Waters HPLC pump and refractive index detector, Polymer Standard Service Suprema columns (3000 and $100 \mathrm{~A}$ ), and a linear PEG standard. All samples for GPC were prepared at a concentration of $0.2 \mathrm{wt} \%$ and filtered through a $0.4 \mu \mathrm{m}$ filter. A mobile phase of $0.1 \mathrm{M}$ sodium nitrate and $0.1 \mathrm{M}$ sodium dibasic phosphate, injection volume of $25 \mathrm{~mL}$, and flow rate of $0.5 \mathrm{~mL} / \mathrm{min}$ were used for all samples.

\section{PEG-Gelatin Network Formation and Mechanical Measurements}

The photoinitiator lithium phenyl-2,4,6-trimethylbenzoylphosphinate (LAP) was synthesized as previously described [38]. Gelatin $(2 \mathrm{wt} \%(w / v))$ functionalized with varying amounts of norbornene was prepared in PBS. We used $2 \mathrm{wt} \%$ solutions since this is the critical chain overlap concentration above which gelatin can form physical gels at room temperature [39]. The gelatin macromolecules were crosslinked by $0.1 \mathrm{wt} \%(0.3 \mathrm{mM}) 3.5 \mathrm{kDa}$ PEG dithiol (JenKem Technology) via a photoinitiated thiol-ene polymerization with $0.05 \mathrm{wt} \%(1.7 \mathrm{mM})$ LAP and light $\left(I_{0}=\sim\right.$ $3.5 \mathrm{~mW} / \mathrm{cm}^{2}, \lambda=365 \mathrm{~nm}$ ) for $30 \mathrm{~s}$. For all experiments, 40 $\mu \mathrm{L}$ cylindrical gels (O.D. $\sim 5 \mathrm{~mm}$, height $\sim 2 \mathrm{~mm}$ ) were formed in the cut end of a $1 \mathrm{~mL}$ syringe (BD Medical).

The various gels were placed in cell culture media to swell overnight and weighed the next day. The volume swelling ratio $Q$ was calculated by first solving for the mass swelling ratio $q$, which involved dividing the measured swollen mass by the theoretical dry mass (data not shown) and assuming that the density of the polymer was similar to that of its solvent. Parallel plate rheometry was performed on crosslinked gels that were formed with varied norbornene functionalization on the gelatin using an Ares 4400 DHR-3 shear rheometer (TA instruments) with $10 \%$ strain frequency sweep and a $10 \mathrm{rad} / \mathrm{s}$ strain sweep. The shear modulus $(G)$ of the constructs was determined when the gels were in the linear viscoelastic regime for both the frequency and the strain sweep. The final network crosslinking density $\rho_{x L}$ was calculated from rubber elastic theory [40] where $\rho_{x L}=G Q^{1 / 3}(\mathrm{RT})^{-1}$.

\section{Cell Harvest and Expansion}

Primary chondrocytes were isolated from articular cartilage of the femoral-patellar groove of 6-month-old Yorkshire swine as detailed previously [41]. Cells were grown in a T-75 culture flask with media as previously described [42] and were used directly after expansion, $\mathrm{P} 0$, for all cell-based experiments. Briefly, chondrocytes were grown in growth medium (highglucose Dulbecco's modified Eagle's medium [DMEM] 
supplemented with ITS+ Premix $1 \% v / v$ (BD Biosciences), $50 \mathrm{mg} / \mathrm{mL}$ L-ascorbic acid 2-phosphate, $40 \mu \mathrm{g} / \mathrm{mL}$ L-proline, $0.1 \mu \mathrm{M}$ dexamethasone, $110 \mu \mathrm{g} / \mathrm{mL}$ pyruvate, and $1 \%$ penicillin-streptomycin-fungizone) with the addition of $10 \mathrm{ng} / \mathrm{mL}$ insulin-like growth factor (IGF-1) (Peprotech) to maintain cells in a dedifferentiated state. ITS was used because it can promote formation of articular cartilage from chondrocytes [43]. Cultures were maintained at $5 \% \mathrm{CO}_{2}$ and $37^{\circ} \mathrm{C}$.

\section{Chondrocyte Encapsulation and Viability Assessment}

Chondrocytes were encapsulated at 40 million cells $/ \mathrm{mL}$ in 40 $\mu \mathrm{L}$ cylindrical PEG-gelatin gels using gels formed from the 1:1 NB:gelatin amine synthesis conditions $(\sim 4.5 \mathrm{mM}$ norbornene per gelatin chain). This macromolecule formulation degraded readily in response to chondrocyte-secreted enzymes. Cell-laden gels were immediately placed in $1-\mathrm{mL}$ DMEM growth medium in 48-well nontreated tissue culture plates. Media was changed every 3 days. At days 1, 7, and 14, cell viability and cellularity were assessed using a LIVE/DEAD ${ }^{\circledR}$ (Life Technologies) membrane integrity assay and confocal microscopy with Image $(\mathrm{NIH})$ for assessment of cell circularity.

\section{Biochemical Analysis of Cell-Hydrogel Constructs}

On days 1,7 , and 14 , gels were removed from culture $(n=3)$, weighed directly to determine the wet weight, snap frozen in $\mathrm{LN}_{2}$, and stored at $-70{ }^{\circ} \mathrm{C}$ prior to biochemical analysis. Hydrogels were digested in $500 \mu \mathrm{L}$ enzyme buffer $(125 \mu \mathrm{g} /$ $\mathrm{mL}$ papain [Worthington Biochemical] and $10 \mathrm{mM}$ cysteine) and homogenized using $5 \mathrm{~mm}$ steel beads in a TissueLyser (Qiagen). Homogenized samples were digested overnight at $60{ }^{\circ} \mathrm{C}$.

Digested constructs were analyzed for biochemical content. DNA content, as an indicator of cell number, was measured using a PicoGreen assay (Life Technologies), and the results were expressed as the amount of DNA $(\mu \mathrm{g})$ per chondrocyte-laden gel. Sulfated glycosaminoglycan (sGAG) content was assessed using a dimethylmethylene blue assay as previously described with results presented in equivalents of chondroitin sulfate [44]. As a cell-free control, digested acellular gels were assessed by the colorimetric assays, and the resulting values were subtracted from their respective cellladen sample values. GAG content was expressed as a percentage of the wet weight of the respective gels.

\section{Histology and Immunofluorescence Analysis}

On day 14, constructs $(n=3)$ were fixed in $10 \%$ formalin for $30 \mathrm{~min}$ at RT and then snap frozen and cryosectioned to $20 \mu \mathrm{m}$ sections as previously described [45]. At day 14, sections were stained for Safranin-O and Masson's trichrome on a Leica autostainer XL and imaged in bright field (20x objective) on a Nikon (TE-2000) inverted microscope.

For immunostaining, on day 14, sections were blocked with $5 \%$ BSA and then analyzed by anti-type II collagen (1:50, US Biological) and anti-type I collagen (1:50, Abcam). Sections were pretreated with appropriate enzymes for $1 \mathrm{~h}$ at $37^{\circ} \mathrm{C}$ : hyaluronidase (2080 U) for type II collagen and Pepsin A (4000 U) with Retrievagen A (BD Biosciences) treatment for type I collagen to help expose the antigen. Sections were probed with AlexaFluor 555-conjugated secondary antibodies (1:200, Life Technologies) and counterstained with DAPI to reveal cell nuclei. All samples were processed at the same time to minimize sample-to-sample variation. Images were collected on a Zeiss LSM710 scanning confocal microscope with a $20 \mathrm{X}$ objective using a Z-stack maximum intensity projection of $4 \times 5 \mu \mathrm{m}$ slices of the $20 \mu \mathrm{m}$ section using the same settings and postprocessing for all the images. The background gain was set to negative controls on acellular sections that received the same treatment. Positive controls were performed on porcine hyaline cartilage for type II collagen and porcine meniscus for type I collagen (Supplementary Fig. 2a, b).

\section{Statistical Analysis}

Data are shown as mean \pm standard deviation. One-way ANOVA with a two-tailed Bonferroni's multiple comparison posttest was used to assess differences in experimental outputs at different culture time points, since means of three samples are being compared simultaneously. $\alpha=0.05$ was used for all analysis. Statistical calculations were performed on GraphPad Prism ${ }^{\circledR} . p<0.05$ was considered to be statistically significant.

\section{Results}

\section{Modifying PEG-Gelatin Network Properties}

By varying the extent of norbornene functionalization of gelatin, we aimed to tune the crosslinking density of the final network and therefore the macroscopic properties of the resulting hydrogel. Figure 1a contains a schematic of gelatin functionalization with norbornene, along with the estimated amount of norbornenes attached to each gelatin molecule (based on calculations). Figure $1 b$ depicts the photopolymerization between $2 \mathrm{wt} \% \mathrm{NB}$-functionalized gelatin with varying amounts of norbornene and $0.1 \mathrm{wt} \%$ PEG dithiol along with the concentrations of norbornene and thiol.

We characterized the efficiency of lysine modification on gelatin by the 5-norbornene-2-acetic acid succinimidyl ester reaction using the TNBSA assay, with data shown in Table 1 $(n=5)$. The 1:1 norbornene:gelatin amine condition led to $75 \pm$ 
Fig. 1 Gelatin functionalization with norbornene and

photopolymerization with PEG. a Depiction of the reaction of gelatin with varying molar amounts of norbornene succinimidyl ester. The reaction proceeded for $1 \mathrm{~h}$ at $37^{\circ} \mathrm{C}$ and the amount of functionalization on the product was measured by TNBSA. The estimated amount of norbornenes per gelatin molecule is shown below based on functionalization efficiency calculations. b

Photopolymerization scheme between $2 \mathrm{wt} \%$ norbornenefunctionalized gelatin with varying amounts of norbornene $(1.1-$ $4.5 \mathrm{mM}$ ) and $0.1 \mathrm{wt} \% 3.5 \mathrm{kDa}$ PEG dithiol with $(0.6 \mathrm{mM})$ thiol to form a covalently crosslinked hybrid scaffold



$6 \%$ functionalization efficiency, which is similar to that observed with other studies using similar amine-modifying techniques $[32,33]$. Lower stoichiometric ratios (0.5:1 and 0.25:1 norbornene/gelatin amine) led to corresponding decreases in the functionalization efficiency, $36 \pm 5 \%$ and $19 \pm 5 \%$, respectively. The unmodified gelatin was measured to have 21 amine groups per molecule, which was also confirmed by other studies $[32,36]$, and suggests that the synthesized gelatin macromolecules have on average $17(75 \%), 8(36 \%)$, and $4(19 \%)$ norbornene pendent groups per molecule for crosslinking. The swollen shear modulus of the resulting acellular constructs was measured, and the crosslinking density of the scaffold was calculated. Consistent with the expected crosslinking reaction, both values increased with increasing amount of norbornene functionalization as shown in Table $1(n=5)$.

\section{Verification of Degradation of Modified Gelatin by Chondrocyte-Secreted Enzymes}

Modification of proteins can alter their structure and change their susceptibility to enzymatic degradation since altered lysines may affect the way that an enzyme binds to its site to initiate cleavage. To verify that the modified gelatin could degrade in the response to chondrocyte-secreted enzymes, we performed a solution-based assay and tested the degradability of various modified gelatin macromolecules when exposed to either collagenase or chondrocyte-conditioned media. We chose chondrocyte-conditioned medium that was collected after 3 days of culture, since we have previously observed that the secreted enzymes can cleave a collagenderived peptide sequence during that time frame [15]. After incubation of the modified gelatin with enzymes, GPC was

Table 1 Material properties of acellular hybrid scaffolds $(n=5)$

\begin{tabular}{lllll}
\hline $\begin{array}{l}\text { Molar ratio of norbornene } \\
\begin{array}{l}\text { succinimidyl ester reacted } \\
\text { with gelatin amines }\end{array}\end{array}$ & $\begin{array}{l}\text { Norbornene } \\
\text { functionalization } \\
\text { efficiency }(\%)^{\mathrm{a}}\end{array}$ & $\begin{array}{l}\text { Calculated amount of } \\
\text { norbornene on gelatin } \\
\text { chain }(\mathrm{mM})^{\mathrm{b}}\end{array}$ & $\begin{array}{l}\text { Thiol } \\
\text { concentration } \\
(\mathrm{mM})\end{array}$ & $\begin{array}{l}\text { Swollen shear } \\
\text { modulus } G \\
(\mathrm{kPa})\end{array}$ \\
\hline $\begin{array}{l}\text { Crosslinking } \\
\text { density } \rho_{x L} \\
(\mathrm{mM})^{\mathrm{c}}\end{array}$ & $\sim 1.1$ & 0.6 & 0.6 & $<0.1$ \\
$0.5: 1$ Norbornene:gelatin amines & $36 \pm 5$ & $\sim 2.2$ & 0.18 & 0.6 \\
$1: 1$ Norbornene:gelatin amines & $75 \pm 6$ & $\sim 4.5$ & 0.1 \\
\hline
\end{tabular}

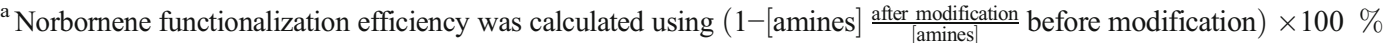

${ }^{\mathrm{b}}$ Norbornene concentration per gelatin molecule was calculated by accounting for the amount of amines on type A gelatin $(\sim 21)$ and the norbornene functionalization efficiency for each condition assuming that only norbornenes replaced the gelatin amines

${ }^{\mathrm{c}}$ Crosslinking density was calculated from the rubber elastic theory: $\rho_{x L}=G Q^{1 / 3}(\mathrm{RT})^{-1}$ 
used to monitor the degradation of both unmodified and norbornene-substituted gelatin. The unmodified gelatin peak elutes at $18 \mathrm{~min}$ and is clearly distinguished from a large system peak at $20 \mathrm{~min}$ that occurs as a consequence of a small dissimilarity in composition, and thus a refractive index difference, between the injected buffer and the mobile phase. A substitution of $0.25: 1$ or $0.5: 1 \mathrm{NB}$ :gelatin amine resulted in no significant change in retention time compared to the unmodified gelatin, while the 1:1 NB:gelatin amine showed a distinguishable yet slightly shifted and significantly smaller peak. We hypothesize that this may be due to the formation of a slightly more hydrophobic macromolecule that may be more susceptible to interaction with the column stationary phase than the unmodified gelatin. When collagenase was added to each gelatin sample, the peak at 18 min completely disappears, indicating degradation of gelatin into smaller molecular weight fragments. A lower MW shoulder appears on the system peak, which corresponds to the gelatin degradation products. Finally, chondrocyte-conditioned medium was added to each gelatin sample to assess degradation in the presence of cell-secreted enzymes. Similar to treatment with collagenase, the gelatin peak was shifted to the low-molecular-weight shoulder, indicating that the norbornene-functionalized gelatin can be degraded by chondrocyte-secreted enzymes. Results are summarized in Supplementary Fig. 1, while Fig. 2 shows the effect of adding collagenase or chondrocyte-secreted enzymes to the most highly substituted gelatin (i.e., reacted at 1:1 NB:gelatin amines).

\section{Chondrocyte Viability, Cellularity, and Morphology}

Gelatin amines that were reacted at a 1:1 molar ratio with norbornene were used in all subsequent cell encapsulation



Fig. 2 GPC chromatogram of gelatin functionalized with norbornene at 1:1 NB:gelatin amine that is either untreated $(A)$ or treated with $20 \mathrm{U} / \mathrm{mL}$ collagenase for $1 \mathrm{~h}$ at $37^{\circ} \mathrm{C}(B)$ or treated with day 3 chondrocyteconditioned media for $1 \mathrm{~h}$ at $37^{\circ} \mathrm{C}(C)$. The untreated gelatin peak that is found at 18 min elution disappears after treatment with either collagenase or chondrocyte-conditioned media suggesting degradation of gelatin to smaller by-products by the enzymes experiments, since this formulation contained a higher amount of covalent crosslinks and thus could retain a gel structure for a longer period of time for the slow-growing chondrocytes. We chose a chondrocyte seeding density of 40 million cells/ $\mathrm{mL}$, as chondrocytes have been studied at this density for cartilage tissue engineering experiments using 3D scaffolds and shown to secrete an elaborate ECM at this concentration [46-48]. After chondrocytes were encapsulated, their viability, cellularity, and morphology were assessed at days 1,7 , and 14. In Fig. 3a, the chondrocytes retained spherical morphology at day 1 and begin to spread as observed on days 7 and 14 , which suggests that the chondrocytes are able to locally remodel the environment. Image processing reveals that the circularity of the chondrocytes decreases from day 1 to day 7 and day 14 as shown in Fig. 3b, further corroborating the results seen in Fig. 3a.

Additionally, the cells retain high viability (calculated to be $>95 \%)$ at all the time points and appear to proliferate over time, as there are many more cells in the constructs at day 14 than there are at day 1 . This increase in cellularity was quantified by the PicoGreen analysis that measures DNA content and shows a significant increase in cell number between day 1 and day $14(p=0.01)$ (Fig. 3c). Collectively, these results suggest that chondrocytes can thrive, proliferate, and remodel their environment in this scaffold system.

\section{ECM Production and Deposition in Scaffolds}

GAG content was assessed biochemically at days 1, 7, and 14 , and the distribution of secreted matrix molecules was examined by staining sections with Safranin-O (GAG) and Masson's trichrome (collagen). Measured quantities of the matrix molecules were normalized to the wet weight of each construct. As Fig. 4a shows, at day 14, GAGs (red) were extensively distributed throughout the gel. Staining of an acellular construct revealed low background staining (Fig. 4b), indicating that staining was primarily from macromolecules secreted by the resident chondrocytes. Furthermore, quantitative analysis, as shown in Fig. 4c, supports the conclusion that the amount of sGAG deposition increased significantly from day 1 to day $14(p=0.0004)$. A similar distribution pattern to what was seen with GAGs is observed with collagen (dark blue) as seen in Fig. 5a, b. Figure 5e depicts the scaffold as optically opaque, with a cartilaginous appearance after 14 days of culture, much more than either the acellular (Fig. 5c) or day 1 time point (Fig. 5d), further suggesting widespread ECM distribution in the scaffold [49]. This versatile PEG-crosslinked gelatin system appears to provide a scaffold that is readily degraded by chondrocytes and can support matrix deposition over time. 
a
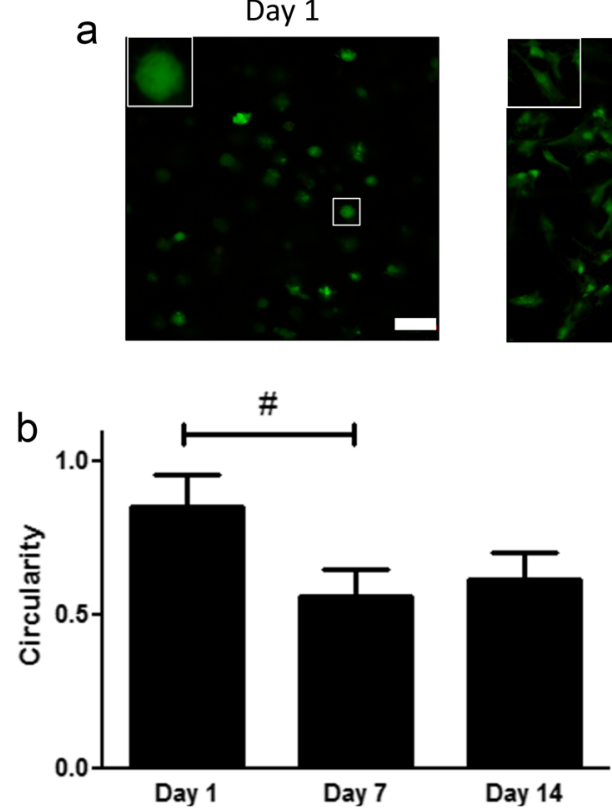

Fig. 3 Viability, morphology, and cellularity of 1:1 NB:gelatin amine hybrid scaffold. a Live (green)/dead (red) stains of encapsulated chondrocytes. Viability is calculated to be greater than $95 \%$ at all the time points. Insets highlight single cells changing in morphology from rounded to more spread over time. Scale bars represent $50 \mu \mathrm{m}$. b Degree of circularity between 0 (not rounded) and 1(rounded) of the encapsulated
Day 7

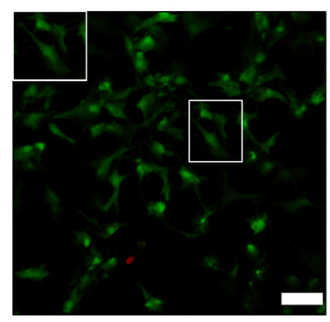

Day 14
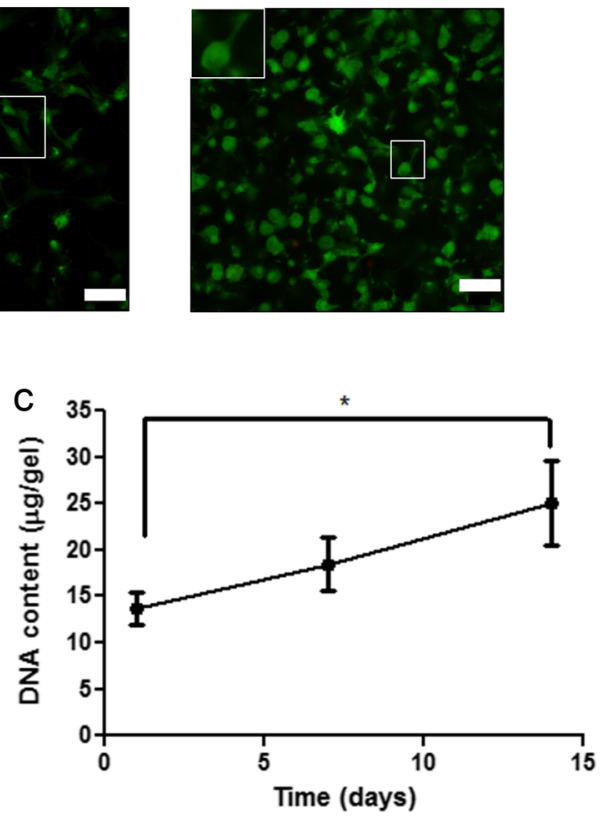

cells. A number sign with a line indicates a statistically significant difference in circularity value between day 1 and day $7(p=0.02)$. Results are presented as mean $\pm \mathrm{SD}(n=3)$. c DNA content ( $\mu \mathrm{g}$ of DNA/ gel) as measured by Picogreen assay over 14 days. An asterisk with a line indicates a statistically significant difference between day 1 and day 14 in DNA content $(p=0.01)$. Results are presented as mean $\pm \mathrm{SD}(n=3)$

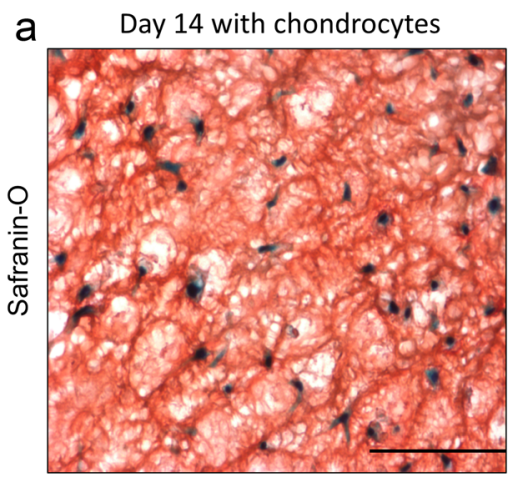

b

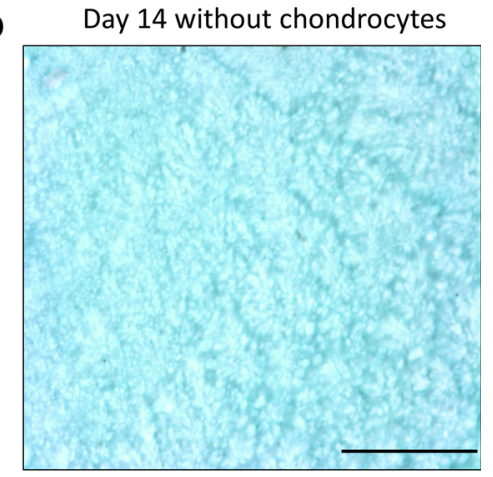

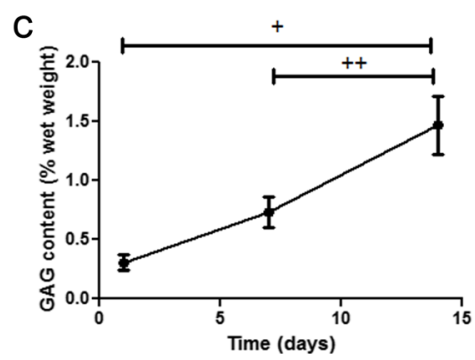

Fig. 4 Glycosaminoglycan distribution and production in a 1:1 NB:gelatin amine hybrid scaffold. a Section with encapsulated chondrocytes at day 14 stained for GAGs with nuclei stained black and GAGs stained red. b Acellular section stained for GAGs at day 14. Scale bars represent $100 \mu \mathrm{m}$. $\mathbf{c}$ Total GAG content expressed as a percentage of the respective construct wet weight assessed at days 1, 7, and 14. A plus sign with a line indicates a statistically significant difference in GAG content between day 1 and day $14(p=0.0004)$, and two plus signs with a line indicates a statistically significant difference in GAG content between day 7 and day $14(p=0.001)$. Results are presented as mean \pm $\mathrm{SD}(n=3)$ 
Fig. 5 Collagen and ECM distribution in a 1:1 NB:gelatin amine hybrid scaffold. a Section with encapsulated chondrocytes at day 14 stained for collagen with nuclei stained violet and collagen stained blue. $\mathbf{b}$ Acellular section stained for collagen at day 14. Scale bars represent $100 \mu \mathrm{m}$. c Gross image of a transparent acellular scaffold at day 1. d Gross image of a translucent scaffold with encapsulated chondrocytes at day 1. e Gross image of an opaque scaffold with encapsulated chondrocytes at day 14 that has a cartilaginous appearance
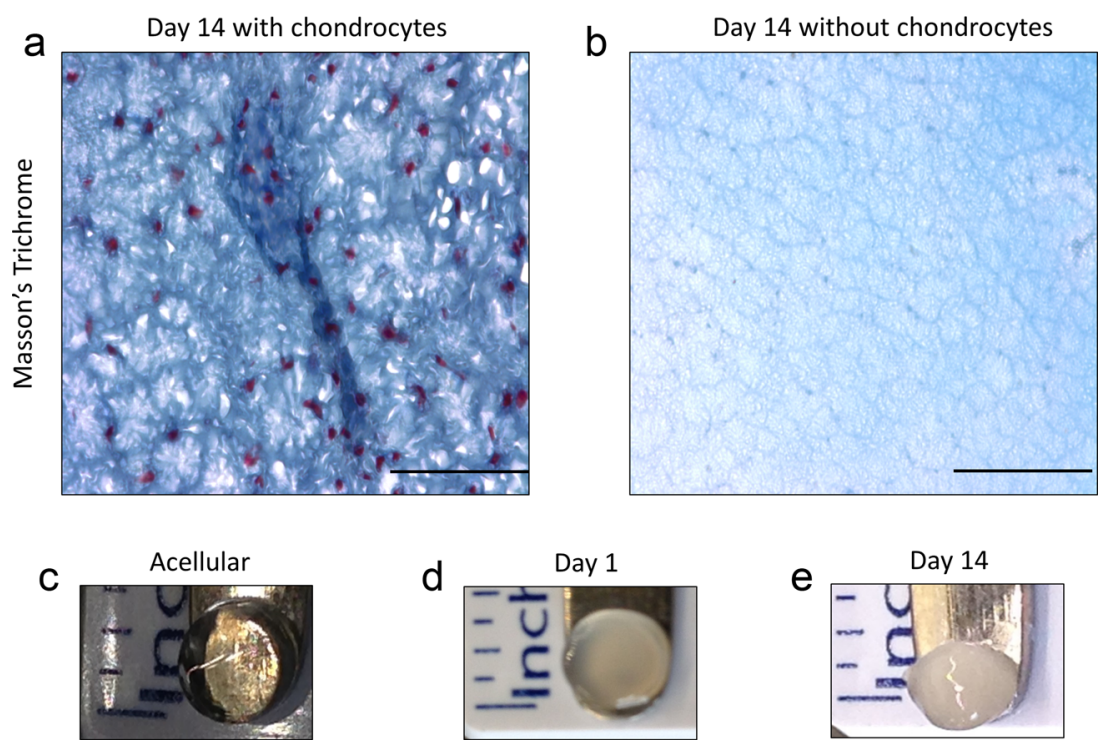

\section{Quality of Collagen Generated by Chondrocytes in the Scaffold}

To verify that the collagen generated by the chondrocytes had an articular cartilage phenotype, we qualitatively assessed the ratio of type II collagen to type I collagen on gel immunostained sections. Images revealed that at day 14, negligible type I collagen (red) was observed, but robust type II collagen (red) was observed throughout the scaffold as seen in Fig. 6.

\section{Discussion}

Engineering a clinically viable scaffold to promote cartilage regeneration is challenging. By using a novel, tunable hybrid biological-synthetic system, we have shown quantitatively and qualitatively, in vitro, that encapsulated chondrocytes



Fig. 6 Type I collagen versus type II collagen distribution assessed by immunofluorescence in a 1:1 NB/gelatin amine hybrid construct with encapsulated chondrocytes at day 14. a Gel section stained for type I collagen. b Gel section stained for type II collagen. Sections were stained generate highly distributed cartilage-specific ECM molecules. We showed that chondrocyte-secreted enzymes can degrade norbornene-functionalized gelatin and further demonstrated that cells are viable when embedded in the hydrogel formulation. The chondrocytes appear to spread throughout the scaffold, suggesting that encapsulated cells are locally remodeling their environment and facilitate the eventual deposition of matrix throughout the scaffold. Future investigations with this scaffold could prove useful in designing a cell carrier system to promote cartilage regeneration in vivo.

Modulus studies confirmed that these PEG-gelatin scaffolds could be tuned to adjust their crosslinking density and macroscopic properties that depend on this parameter (Table 1). In general, it can be more difficult to change material properties of pure protein-based gels, so covalent crosslinking can lead to higher mechanical properties [22]. If the crosslinking density can be adjusted by the user, it can also alter the amount of tissue deposition [9, 50]. Gel properties,

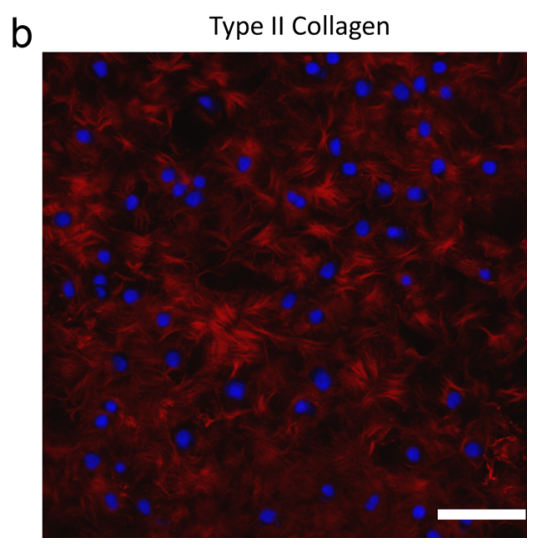

for both anti-collagen type I and anti-collagen type II antibodies (red) and were counterstained with DAPI (blue) for cell nuclei. Scale bars represent $50 \mu \mathrm{m}$ 
degradation, and ECM deposition might be further tuned by changing the network connectivity using different molecular weight PEG crosslinkers or multiarm PEG crosslinkers depending on whether a more tightly or loosely crosslinked network is desired.

We confirmed that chondrocyte-secreted enzymes could degrade norbornene-functionalized gelatin by visualizing the degradation products with GPC (Fig. 2). GPC data suggest that norbornene-functionalized gelatin is degraded to smaller molecular weight products in the presence of chondrocyte-conditioned media. This confirms that norbornene-functionalized gelatin is a viable platform for the formation of chondrocyte-specific cellularly degradable hydrogels. We demonstrated these results in a solution-based assay, but a complementary study has shown that functionalized and crosslinked gelatin can be degraded by cell-secreted enzymes as well [51]. Here, we observe this indirectly by changes in the chondrocyte morphology (Fig. 3a); however, there are alternate ways to design systems that might respond to chondrocyte-secreted enzymes. One idea would be to include an aggrecanase-based cleavable peptide linker [52]. We selected the full-length protein gelatin for this study, as we hypothesized that its multiple MMP-cleavable sites would lead to more facile cleavage by chondrocytes than small peptide sequences as suggested by other groups [18]. In fact, the gelatin molecule contains about six MMP-sensitive sequences per molecule [27], which appears to be readily cleaved by chondrocytesecreted enzymes at the cell density studied.

Viability results revealed that chondrocytes thrive and proliferate in the hybrid network. Cellularity in the gel quantitatively increased, which was confirmed by PicoGreen, and the cells spread over time (Fig. 3). Part of the reason for this change in cell morphology is likely because the chondrocytes are binding to adhesion factors present on the protein [26]. Another explanation is that the cells are degrading the network by a cell-mediated mechanism and spreading [51], which could facilitate the observed widespread matrix deposition. Future studies could focus on improving methods to verify that local degradation is occurring, which could include monitoring the cell-based degradation using microrheological techniques, such as microparticle tracking [53].

Cartilage-specific matrix produced by chondrocytes was distributed throughout the entire scaffold as it was in other cell-mediated degradable scaffolds [15] (Figs. 4a and 5a). Additionally, the GAG production from this scaffold was comparable to what was seen in a cellularly degradable cartilage tissue engineering scaffold with a similar cell seeding density [15]; however, this system did not need to tether a growth factor, such as TGF- $\beta$, to the network in order to elicit a response from chondrocytes. A possible explanation for the robust secretory properties might relate to the fact that gelatin can bind to growth factors released by cells and perhaps present them to embedded chondrocytes in a local and sustained manner [1].
The encapsulated chondrocytes have a lower circularity at day 14 , which can be suggestive of a hypertrophic phenotype that generate higher amounts of type I collagen and functionally inferior cartilage tissue [54]. Interestingly, the collagen produced by the encapsulated cells maintained a higher quality articular cartilage phenotype, as indicated by the collagen typing result with a high type II collagen:type I collagen ratio (Fig. 6).

In a potential clinical application as a scaffold, this system could be advantageous since it is easy to tune formation of this network. Currently, collagen-based materials are used as scaffolds in the clinic and have yielded variable success, partly due to high variations in network formation [55]. The hybrid system presented in this manuscript can be easily tuned and permits the widespread elaboration of cartilage-specific ECM molecules over a short period of time. It would be interesting to see how matrix production is affected as the length of culture time is extended, especially in an in vivo environment where the presence of macrophages could help stimulate chondrocyte gelatinase secretion [28].

\section{Conclusions}

In summary, a novel hydrogel system based on crosslinking a full-length protein, gelatin, by PEG was designed to increase control over network formation and permit local chondrocytemediated degradation for cartilage tissue engineering applications. The hydrogel increases chondrocyte cellularity and facilitates cartilage ECM production via cell-mediated degradation in a manner that promotes widespread matrix deposition in just 14 days. The materials' approach was to modify gelatin with norbornene functionalities that were crosslinked with PEG dithiols via a photoinitiated thiol-ene reaction. Results confirmed that the final PEG-gelatin gel properties could be altered by modifying the amount of norbornene functionalization, while maintaining susceptibility to enzymatic degradation. Culture of chondrocyte-laden hydrogel scaffolds led to ECM molecules distributed throughout the construct and resembled articular cartilage with respect to gross appearance of the generated matrix molecules and collagen typing (high type II collagen:type I collagen ratio). This biosynthetic system may prove useful in clinical applications as a scaffold to promote cartilage regeneration.

Acknowledgments The authors acknowledge Amanda Meppelink for providing knees on which chondrocyte isolations were performed as well as Kathryn Morrissey for help with setting up GPC experiments. The authors would also like to thank Jason Silver, Dr. William Wan, and Dr. Malar Azagarsamy for assistance on experimental design. Funding for these studies was provided by the Howard Hughes Medical Institute, National Institutes of Health (R01 DE016523-10), and Department of Defense (W81XWH-10-1-0791). The US Army Medical Research 
Acquisition Activity, 820 Chandler Street, Fort Detrick, MD, 217025014, USA, is the awarding and administering acquisition office.

Conflicts of Interest The authors have no conflicts of interest to declare.

\section{References}

1. Adolphe M. Biological regulation of the chondrocytes. Boca Raton: CRC Press; 1992.

2. Kim IL, Mauck RL, Burdick JA. Hydrogel design for cartilage tissue engineering: a case study with hyaluronic acid. Biomaterials. 2011;32:8771-82.

3. Hutson CB, Nichol JW, Aubin H, Bae H, Yamanlar S, Al-Haque S, et al. Synthesis and characterization of tunable poly(ethylene glycol): gelatin methacrylate composite hydrogels. Tissue Eng A. 2011;17:1713-23.

4. Kock L, van Donkelaar CC, Ito K. Tissue engineering of functional articular cartilage: the current status. Cell Tissue Res. 2012;347: 613-27.

5. Spiller KL, Maher SA, Lowman AM. Hydrogels for the repair of articular cartilage defects. Tissue Eng B. 2011;17:281-99.

6. Forsyth CB, Cole A, Murphy G, Bienias JL, Im H-J, Loeser RF. Increased matrix metalloproteinase- 13 production with aging by human articular chondrocytes in response to catabolic stimuli. J Gerontol A Biol Sci Med Sci. 2005;60:1118-24.

7. Nicodemus GD, Skaalure SC, Bryant SJ. Gel structure has an impact on pericellular and extracellular matrix deposition, which subsequently alters metabolic activities in chondrocyte-laden PEG hydrogels. Acta Biomater. 2011;7:492-504.

8. Sridhar BV, Doyle NR, Randolph MA, Anseth KS. Covalently tethered TGF- $\beta 1$ with encapsulated chondrocytes in a PEG hydrogel system enhances extracellular matrix production. J Biomed Mater Res A. 2014;102:4464-72.

9. Bryant SJ, Anseth KS. Hydrogel properties influence ECM production by chondrocytes photoencapsulated in poly(ethylene glycol) hydrogels. J Biomed Mater Res A. 2002;59:63-72.

10. Lee KY, Mooney DJ. Hydrogels for Tissue Engineering. Chem Rev. 2001;101:1869-80.

11. Lutolf MP, Hubbell JA. Synthetic biomaterials as instructive extracellular microenvironments for morphogenesis in tissue engineering. Nat Biotechnol. 2005;23:47-55.

12. Bryant SJ, Anseth KS. Controlling the spatial distribution of ECM components in degradable PEG hydrogels for tissue engineering cartilage. J Biomed Mater Res A. 2003;64:70-9.

13. Zhao W, Jin X, Cong Y, Liu Y, Fu J. Degradable natural polymer hydrogels for articular cartilage tissue engineering. J Chem Technol Biotechnol. 2013;88:327-39.

14. Kretlow JD, Mikos AG. From material to tissue: biomaterial development, scaffold fabrication, and tissue engineering. AIChE J. 2008;54:3048-67.

15. Sridhar BV, Brock JL, Silver JS, Leight JL, Randolph MA, Anseth KS. Development of a cellularly degradable PEG hydrogel to promote articular cartilage extracellular matrix deposition. Adv Healthc Mater. 2015;4:635-781.

16. Patterson J, Hubbell JA. Enhanced proteolytic degradation of molecularly engineered PEG hydrogels in response to MMP-1 and MMP-2. Biomaterials. 2010;31:7836-45.

17. Lin Z, Willers $\mathrm{C}, \mathrm{Xu}$ J, Zheng M-H. The chondrocyte: biology and clinical application. Tissue Eng. 2006;12:1971-84.

18. Fields GB, Van Wart HE, Birkedal-hansent H. Sequence specificity of human skin fibroblast collagenase: evidence for the role of collagen structure in determining the collagenase cleavage site. $\mathrm{J}$ Biol Chem. 1987;262:6221-6.

19. Schmidt O, Mizrahi J, Elisseeff J, Seliktar D. Immobilized fibrinogen in PEG hydrogels does not improve chondrocyte-mediated matrix deposition in response to mechanical stimulation. Biotechnol Bioeng. 2006;95:1061-9.

20. Almany L, Seliktar D. Biosynthetic hydrogel scaffolds made from fibrinogen and polyethylene glycol for $3 \mathrm{D}$ cell cultures. Biomaterials. 2005;26:2467-77.

21. Mironi-Harpaz I, Wang DY, Venkatraman S, Seliktar D. Photopolymerization of cell-encapsulating hydrogels: crosslinking efficiency versus cytotoxicity. Acta Biomater. 2012;8:1838-48.

22. Gonen-Wadmany M, Oss-Ronen L, Seliktar D. Protein-polymer conjugates for forming photopolymerizable biomimetic hydrogels for tissue engineering. Biomaterials. 2007;28:3876-86.

23. Appelman TP, Mizrahi J, Elisseeff JH, Seliktar D. The influence of biological motifs and dynamic mechanical stimulation in hydrogel scaffold systems on the phenotype of chondrocytes. Biomaterials. 2011;32:1508-16.

24. Singh RK, Seliktar D, Putnam AJ. Capillary morphogenesis in PEG-collagen hydrogels. Biomaterials. 2013;34:9331-40.

25. Manicourt DH, Devogelaer JP, Thonar EJ. Products of cartilage metabolism. In: Seibel M, Robins SP, Bilezikian JP, editors. Dynamics of bone and cartilage metabolism. 2nd ed. Burlington: Elsevier Science; 2006. p. 421-39.

26. Rice JJ, Martino MM, De Laporte L, Tortelli F, Briquez PS, Hubbell JA. Engineering the regenerative microenvironment with biomaterials. Adv Healthc Mater. 2013;2:57-71.

27. Gorgieva S, Kokol V. Collagen-vs. gelatine-based biomaterials and their biocompatibility: review and perspectives. In: Pignatello R, editor. Biomaterials applications for nanomedicine. Croatia: InTech, 2011; p. 17-52.

28. Dreier R, Wallace S, Fuchs S, Bruckner P, Grassel S. Paracrine interactions of chondrocytes and macrophages in cartilage degradation: articular chondrocytes provide factors that activate macrophage-derived pro-gelatinase B (pro-MMP-9). J Cell Sci. 2001;114:3813-22.

29. Mohtai M, Smith RL, Schurman DJ, Tsuji Y, Torti FM, Hutchinson NI, et al. Expression of 92-kD type IV collagenase/gelatinase (gelatinase B) in osteoarthritic cartilage and its induction in normal human articular cartilage by interleukin 1. J Clin Invest. 1993;92: 179-85.

30. Wang C-C, Yang K-C, Lin K-H, Wu C-C, Liu Y-L, Lin F-H, et al. A biomimetic honeycomb-like scaffold prepared by flow-focusing technology for cartilage regeneration. Biotechnol Bioeng. 2014;111:2338-48.

31. Mazaki T, Shiozaki Y, Yamane K, Yoshida A, Nakamura M, Yoshida Y, et al. A novel, visible light-induced, rapidly crosslinkable gelatin scaffold for osteochondral tissue engineering. Sci Rep. 2014;4:4457.

32. Fu Y, Xu K, Zheng X, Giacomin AJ, Mix AW, Kao WJ. 3D cell entrapment in crosslinked thiolated gelatin-poly(ethylene glycol) diacrylate hydrogels. Biomaterials. 2012;33:48-58.

33. Daniele MA, Adams AA, Naciri J, North SH, Ligler FS. Interpenetrating networks based on gelatin methacrylamide and PEG formed using concurrent thiol click chemistries for hydrogel tissue engineering scaffolds. Biomaterials. 2014;35:1845-56.

34. Mũnoz Z, Shih H, Lin C-C. Gelatin hydrogels formed by orthogonal thiol-norbornene photochemistry for cell encapsulation. Biomater Sci. 2014;2:1063.

35. Hoyle CE, Bowman CN. Thiol-ene click chemistry. Angew Chem Int Ed Engl. 2010;49:1540-73.

36. Einerson NJ, Stevens KR, Kao WJ. Synthesis and physicochemical analysis of gelatin-based hydrogels for drug carrier matrices. Biomaterials. 2003;24:509-23. 
37. Waldeck H, Kao WJ. Effect of the addition of a labile gelatin component on the degradation and solute release kinetics of a stable PEG hydrogel. J Biomater Sci Polym Ed. 2011;23:1595-611.

38. Fairbanks BD, Schwartz MP, Bowman CN, Anseth KS. Photoinitiated polymerization of PEG-diacrylate with lithium phenyl-2,4,6-trimethylbenzoylphosphinate: polymerization rate and cytocompatibility. Biomaterials. 2009;30:6702-7.

39. Mohanty B, Bohidar HB. Microscopic structure of gelatin coacervates. Int J Biol Macromol. 2005;36:39-46.

40. Bryant SJ, Anseth KS. Photopolymerization of hydrogel scaffolds. In: Ma PX, Ellisseeff J, editors. Scaffolding in tissue engineering. Boca Raton: CRC Press; 2006. p. 1-45.

41. Yoo JJ, Bichara DA, Zhao X, Randolph MA, Gill TJ. Implantassisted meniscal repair in vivo using a chondrocyte-seeded flexible PLGA scaffold. J Biomed Mater Res A. 2011;99:102-8.

42. Byers BA, Mauck RL, Chiang IE, Tuan RS. Transient exposure to transforming growth factor beta 3 under serum-free conditions enhances the biomechanical and biochemical maturation of tissue-engineered cartilage. Tissue Eng A. 2008;14: 1821-34.

43. Chua KH, Aminuddin BS, Fuzina NH, Ruszymah BHI. Insulin-transferrin-selenium prevent human chondrocyte dedifferentiation and promote the formation of high quality tissue engineered human hyaline cartilage. Eur Cell Mater. 2005;9:58-67.

44. Farndale RW, Sayers CA, Barrett AJ. A direct spectrophotometric microassay for sulfated glycosaminoglycans in cartilage cultures. Connect Tissue Res. 1982;9:247-8.

45. Ruan J-L, Tulloch NL, Muskheli V, Genova EE, Mariner PD, Anseth KS, et al. An improved cryosection method for polyethylene glycol hydrogels used in tissue engineering. Tissue Eng Part C Methods. 2013;19:794-801.
46. Silverman RP, Passaretti D, Huang W, Randolph MA, Yaremchuk MJ. Injectable tissue-engineered cartilage using a fibrin glue polymer. Plast Reconstr Surg. 1999;103:1809-18.

47. Passaretti D, Silverman RP, Huang W, Kirchhoff CH, Ashiku S, Randolph MA, et al. Cultured chondrocytes produce injectable tissue-engineered cartilage in hydrogel polymer. Tissue Eng. 2001;7:805-15.

48. Ibusuki S, Papadopoulos A, Ranka M, Halbesma G, Randolph M, Redmond R, et al. Engineering cartilage in a photochemically crosslinked collagen gel. J Knee Surg. 2010;22:72-81.

49. Gu Y, Chen P, Yang Y, Shi K, Wang Y, Zhu W, et al. Chondrogenesis of myoblasts in biodegradable poly-lactide-coglycolide scaffolds. Mol Med Rep. 2013;7:1003-9.

50. Bryant SJ, Chowdhury TT, Lee DA, Bader DL, Anseth KS. Crosslinking density influences chondrocyte metabolism in dynamically loaded photocrosslinked poly(ethylene glycol) hydrogels. Ann Biomed Eng. 2004;32:407-17.

51. Benton JA, DeForest CA, Vivekanandan V, Anseth KS. Photocrosslinking of gelatin macromers to synthesize porous hydrogels that promote valvular interstitial cell function. Tissue Eng A. 2009;15:3221-30.

52. Skaalure SC, Chu S, Bryant SJ. An enzyme-sensitive peg hydrogel based on aggrecan catabolism for cartilage tissue engineering. Adv Healthc Mater. 2014;4:420-31.

53. Schultz KM, Anseth KS. Monitoring degradation of matrix metalloproteinases-cleavable PEG hydrogels via multiple particle tracking microrheology. Soft Matter. 2013;9:1570.

54. Huey D, Hu J, Athanasiou K. Unlike bone, cartilage regeneration remains elusive. Science. 2012;6933:917-21.

55. Kon E, Verdonk P, Condello V, Delcogliano M, Dhollander A, Filardo G, et al. Matrix-assisted autologous chondrocyte transplantation for the repair of cartilage defects of the knee: systematic clinical data review and study quality analysis. Am J Sports Med. 2009;37:1565-665. 